\title{
Antenna Grouping Assisted Spatial Modulation for mmWave-based UAV-BS
}

\author{
Xingxuan Zuo ${ }^{1}$, Student Member, IEEE, Jiankang Zhang ${ }^{2}$, Senior Member, IEEE, Gangtao Han ${ }^{1}$, Xiaomin $\mathrm{Mu}^{1}$ \\ 1. School of Information Engineering, Zhengzhou University, Zhengzhou, 450001, China. \\ 2. Department of Computing \& Informatics, Bournemouth University, BH12 5BB, U.K.
}

\begin{abstract}
The flexible deployment without new infrastructure makes unmanned aerial vehicles employing as base stations (UAV-BS) promising for many application. Since the signals in millimeter-wave frequencies have very small wavelengths, large antenna arrays can be placed in the UAV-BS. Thus, the UAV-BS is capable of providing abundant spatial resources. Spatial modulation (SM) is an effective technology in exploiting additional capacity of the spatial domain by transmitting antenna indices as virtual bits information. However, a limitation of the classical SM is a single transmit antenna activated at each time slot. As a result, the multiplexing gain offered by the multiple transmit antennas has a significantly loss. Generalised spatial modulation (GSM) allows several antennas to be activated to overcome the problem of SM. However, GSM has a improvement in throughput, while suffers from the performance loss resulting from the channel correlation, which is generated by multiple active antennas. Thus, the grouping SM (GrSM) is utilized to offer spatial capacity for the UAV-BS in mmWave frequency. Specially, the transmit antennas of the UAV-BS are partitioned into groups based on their channel characteristics. The SM is adopted by each group, and the multiplexing gain is achieved across groups. Moreover, the deployment of the UAV-BS has significantly influence on the throughput of the system. In this paper, we formulate a problem to maximize the achievable sum rate of the ground user. The GrSM scheme is utilized to obtain extra throughput in spatial domain. Since the dimension of the UAV position is not very high, a grid based exhaustive search method is adopted to solve the optimization problem. Simulation results demonstrate the proposed solution has an improvement in terms of the sum rate performance.
\end{abstract}

Index Terms-unmanned aerial vehicle(UAV), millimeter-wave (mmWave), deployment, generalised spatial modulation (GSM), antenna grouping, achievable sum rate

\section{INTRODUCTION}

Unmanned aerial vehicles (UAVs) have drawn a lot of attentions both in military and in civilian societies due to their flexible mobility and low cost [1]. UAVs acted as base stations (UAV-BSs) are capable of quickly deploying without terrestrial infrastructures, and thereby are applicable to the urgent and temporal communications [2]. The utilization of millimeterwave (mmWave) technology enables large antenna arrays to be packed in small dimension according to its wavelength [3]. Hence, mmWave-based UAV-BSs provide more benefits, such as sufficient bandwidth and larger capacity [4]. Considering large antenna arrays are deployed in the UAV-BS, UAV-BS mmWave systems have abundant spatial resources.

In spatial modulation (SM), the information bits consist$\mathrm{s}$ of the constellation point by conveying amplitude/phase modulation (APM) and the antenna indices using space shift keying (SSK) modulation [5]. SM is an effective technique in exploiting the spatial resources by delivering virtual bit information using antenna indices. However, since a single transmit antenna is activated at each time slot, the data rate of SM is limited [6]. Generalised spatial modulation is to alleviate the limitation of SM, where more than one transmit antennas are activated at each time slot [7]. GSM techniques can significantly enhance the spectral efficiency, but it suffers from inter-channel interference at the receiver. As investigated by Kim et. al. [8] and our previous investigation [8], antenna grouping exploiting channel correlation is able both to degrade the loss of multiplexing gain and to improve the achievable capacity. Thus, the grouped SM (GrSM) [8], [9] has been proposed by partitioning the transmit antennas into several groups according to different criteria.

In particular, UAVs are promising to enhance the performance of the existing wireless network [4]. Since the channel gain between UAVs and the ground users can be obtained with the location information [10]. The deployment and placement optimization of the UAV have been studied. With fixed altitude of the UAV-BSs, the horizontal coordinates have been adjusted to cover all ground users with minimum number of required UAV-BSs [11]. In [12], the deployment of several UAV-BSs has been studied to cover a maximum number of users in a target area. A 3D beamforming approach utilizing coordinate transformation has been investigated to achieve flexible coverage for UAV-BS in mmWave frequency [13]. In [14], the optimization of the deployment and the beamforming design for the UAV-BS in mmWave bands has been analysed in order to achieve the a maximum sum rate.

Against this background, we aim to maximize the achievable sum rate the mmWave-based UAV-BS relying on GrSM scheme. More specially, the transmit antennas of the UAV-BS are partitioned into several groups according to their channel correlation. Furthermore, SM is independently utilized by each group and hence the multiplexing gain can be achieved across groups. Thus, the sum rate includes two parts. One is in the classic signal domain, the other one is in the spatial domain. Intuitively, there is no closed-form solutions for the optimization problem. Luckily, the dimension of the UAV-BS positions is not very high, hence, in this paper, we rely on grid search algorithm in obtaining the optimal UAV-BS location.

The rest of this paper is organized as follows. The mmWavebased UAV-BS system model and the GrSM scheme used are described in Section II. Section III is devoted to the 


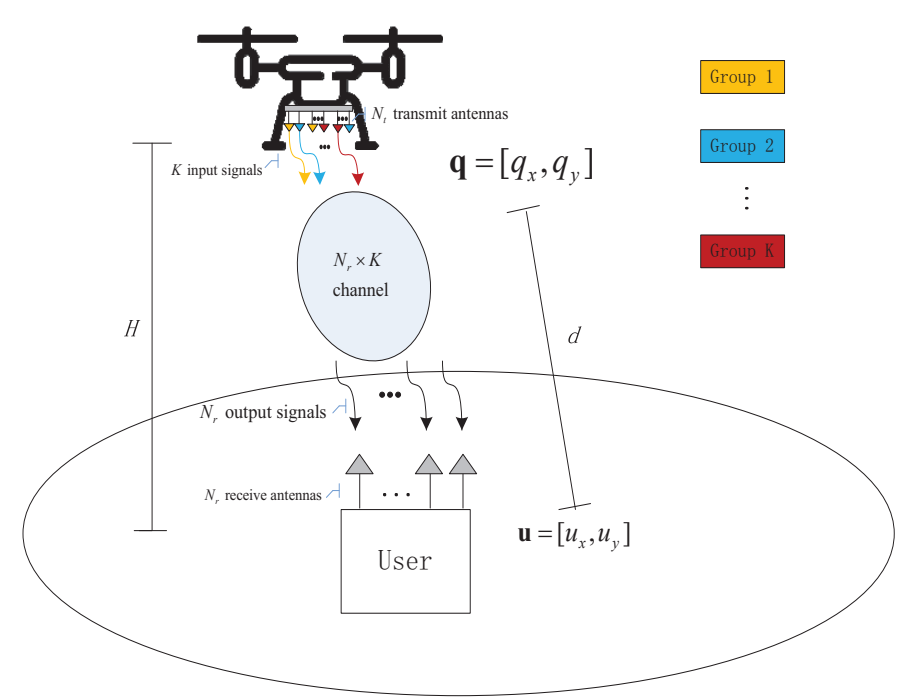

Fig. 1. System model of grouping spatial modulation

analysis of sum rate achieved by the mm-Wave-based UAVBS communication system. Furthermore, grid search algorithm is then presented in Section IV. Our simulation results and discussions are presented in Section V. At last, our conclusions are offered in Section VI.

\section{System ModeL}

As shows in Fig. 1, a rotary-wing mmWave-based UAV-BS communicates with a ground user, where the UAV-BS employs $N_{t}$ transmit antennas and the ground user is equipped with $N_{r}$ receive antennas. The UAV-BS utilizes the GrSM scheme, which activates $K\left(1 \leq K \leq N_{t}\right)$ RF chains for downlink transmission. Furthermore, a 3D Cartesian coordinate system is considered in this paper, and the horizontal coordinate of the ground user is defined as $\boldsymbol{u}=\left[u_{x}, u_{y}\right]^{T}$. The UAV-BS is flying at altitude of $H_{U}$, and the horizontal location of UAV-BS projected on the ground is $\boldsymbol{q}=\left[q_{x}, q_{y}\right]^{T}$. Thus, the distance between the UAV-BS and the ground user is given by $d=$ $\sqrt{H_{U}^{2}+\|\boldsymbol{q}-\boldsymbol{u}\|^{2}}$.

\section{A. Path loss}

Since the scattering is limited in the mmWave band [13], we assume that the wireless links from the UAV-BS to the ground user are dominated by line of sight (LoS). Thus, the path loss of our system can be formulated as [15],

$$
L_{L o S}=32.4+20 \log _{10}\left(f_{m m}\right)+20 \log _{10}(d)
$$

where $f_{m m}[\mathrm{GHz}]$ is the carrier frequency. Typically, the mmWave frequency $f_{m m}=28 \mathrm{GHz}$ will be used for mmWave communications [16]. Hence, the path loss between the UAV$\mathrm{BS}$ and the ground user can be rewritten as,

$$
L_{L o S}=\alpha_{L}+20 \log _{10}(d)
$$

where $\alpha_{L}=32.4+20 \log _{10}\left(f_{m m}\right)$. Let $C_{m m}=10^{\alpha_{L} / 10}$, the received signal power of the ground user can be expressed as,

$$
\begin{aligned}
P_{r} & =P_{t} \cdot 10^{-L_{\text {LoS }} / 10} \\
& =\frac{P_{t}}{C_{m m}\left(H_{U}^{2}+\|\boldsymbol{q}-\boldsymbol{u}\|^{2}\right)}
\end{aligned}
$$

where $P_{t}$ is the transmission power of the UAV-BS.

\section{B. Grouping Spatial Modulation}

For GrSM scheme, the transmit antennas are partitioned into several groups according to their channel correlation. Furthermore, a single antenna from each group is activated for transmitting an APM signal. Thus, the antennas from multiple groups concurrently transmit the APM signals and the SSK symbols.

Explicitly, the total $N_{t}$ transmit antennas are equally divided into $K$ groups, where $K$ is a common divisor of $N_{t}$. Thus, each group has $N_{g}=\frac{N_{t}}{K}$ antennas. We assume that each antenna has the same probability to be activated. Since each group has a single activated antenna, the number of antenna patterns for the GrSM scheme is $\left(\begin{array}{c}N_{g} \\ 1\end{array}\right)$, where $(\cdot)$ represents binomial operation. By contrast, GSM scheme activates multiple antennas simultaneously, and thereby the number of antenna combinations for the GSM scheme is $\left(\begin{array}{c}N_{t} \\ K\end{array}\right)$.

\section{The Received Signal Model}

With the LoS path loss in mmWave frequencies, the received signal can be formulated as [17],

$$
\boldsymbol{y}=\sqrt{P_{r}} \boldsymbol{H} \boldsymbol{x}+\boldsymbol{n}=\sqrt{P_{r}} \sum_{n=1}^{N_{r}} \boldsymbol{H}_{n} e_{s_{n}} x_{n}+\boldsymbol{w}
$$

where $\boldsymbol{H} \in \mathbb{C}^{N_{r} \times N_{t}} . \boldsymbol{w}=\left[w_{1}, w_{2}, \ldots, w_{N_{r}}\right]^{T} \in \mathbb{C}^{N_{r} \times 1}$ is the additive white Gaussian noise (AWGN), $w_{i}$ is with zero mean and variance $\sigma_{w}^{2}$. Since each group have $N_{g}$ transmit antennas, $\boldsymbol{x}$ can be expressed as $\boldsymbol{x}=\left[e_{s_{1}}^{T} x_{1}, e_{s_{2}}^{T} x_{2}, \cdots, e_{s_{K}}^{T} x_{K}\right]^{T}$, and $x$ also can be written as,

$$
\boldsymbol{x}=[\underbrace{0,0, \cdots, x_{1}, \cdots, 0}_{N_{g}}, \underbrace{0, x_{2}, 0, \cdots, 0}_{N_{g}}, \cdots, \underbrace{0, \cdots, 0, x_{K}}_{N_{g}}]^{T}
$$

where $s_{k}$ is the SSK symbol which activates one of the $N_{g}$ transmit antennas to transmit an APM symbol $x_{k}$.

Furthermore, the channel matrix between the UAV-BS and the ground user can be expressed as [18],

$$
\boldsymbol{H}=\boldsymbol{R}_{\mathrm{rx}}^{\frac{1}{2}} \tilde{\boldsymbol{H}} \boldsymbol{R}_{\mathrm{tx}}^{\frac{1}{2}}
$$

where the element of $\tilde{\boldsymbol{H}} \in \mathbb{C}^{N_{r} \times N_{t}}$ is independently identically distributed (i.i.d) complex Gaussian random variable, and has the distribution of $\mathcal{C N}(0,1) . \boldsymbol{R}_{\mathrm{rx}}$ and $\boldsymbol{R}_{\mathrm{tx}}$ are the correlation matrices for the receive antennas and transmit antennas, respectively. Specifically, the exponential correlation model is adopted [19], and $\boldsymbol{R}_{\mathrm{rx}}$ and $\boldsymbol{R}_{\mathrm{tx}}$ can be detailed as,

$$
\boldsymbol{R}_{\mathrm{rx} / \mathrm{tx}}=\left[\begin{array}{ccccc}
1 & \gamma & \gamma^{2} & \cdots & \gamma^{N-1} \\
\vdots & \ddots & \ddots & \ddots & \vdots \\
\gamma^{N-1} & \cdots & \gamma^{2} & \gamma & 1
\end{array}\right]
$$


where $\gamma=\exp (-\beta)$, and $\beta$ is the correlation coefficient between two adjacent antennas. $N$ is the number of transmit/receive antennas.

\section{Antenna Grouping Scheme}

In order to maximize the achievable transmission rate of the UAV-BS, the normalized instantaneous channel correlation matrix (NICCM) is adopted to group the transmit antennas. Specifically, the NICCM is defined as [20],

$$
\boldsymbol{M}=\left[\begin{array}{cccc}
1 & m_{1,2} & \cdots & m_{1, N_{t}} \\
m_{2,1} & 1 & \cdots & m_{2, N_{t}} \\
\vdots & \vdots & \ddots & \vdots \\
m_{N_{t}, 1} & m_{N_{t}, 2} & \cdots & 1
\end{array}\right]
$$

and the $(i, j)$-th element of $\boldsymbol{M}$ is calculated as,

$$
\boldsymbol{M}_{i j}=\frac{1}{\left\|\boldsymbol{h}_{i}\right\| \cdot\left\|\boldsymbol{h}_{j}\right\|} \cdot\left[\boldsymbol{H}^{\mathrm{H}} \boldsymbol{H}\right]_{i j}
$$

where $\boldsymbol{h}_{i}$ is the $i$-th column vector of $\boldsymbol{H} .\left[\boldsymbol{H}^{\mathrm{H}} \boldsymbol{H}\right]_{i j}$ is $i$ th row and $j$-th column element in matrix $\boldsymbol{H}^{\mathrm{H}} \boldsymbol{H}$, and $m_{j, i}$ is the complex conjugate of $m_{i, j}$. Moreover, $M_{i, j}$ denotes the correlation between the channels from the $i$-th transmit antenna and that from the $j$-th transmit antenna. A bigger value of $\left|m_{i, j}\right|$ implies a stronger correlation between the corresponding pair of antennas.

Since the multiplexing gain decreases with the increase of the antenna correlation [18], to achieve the sum rate of the UAV-BS, the $N_{t}$ antennas are partitioned into $K$ disjoint subsets. The antennas having stronger correlation are classified into same group, and then SM is applied intra-group antennas, while the transmit antennas assigned to different group are as weak correlated as possible. In this way, the multiplexing gain relying on the transmit antennas from different groups can be maximized. The SSK symbols can be easily detected by maximum likelihood detection, since the signals from different groups are generally different.

\section{OPTIMIZATION PROBLEM FOR UAV WITH GRSM}

Since the GrSM scheme is adopted in the mmWave UAV-BS system, the number of bits conveyed per symbol includes the information bits transmitted by APM and that conveyed by the SSK. Hence, the total sum-rate per symbol can be expressed as [21],

$$
R_{\text {sum }}=R_{\text {signal }}+R_{\text {spatial }}
$$

where $R_{\text {signal }}$ and $R_{\text {spatial }}$ represent the rate transmitted by the signal symbols and that by the spatial symbols, respectively.

We assume that the transmit signal is normalized to have unit average power, meaning that $E_{s}=E\left[\|x\|^{2}\right]=1$. We firstly focus on the first item in Eq. (10). With a given channel $\boldsymbol{H}$, if the antenna pattern is determined, for example, the $i$-th antenna pattern is given, the achievable rate can be expressed as [22],

$$
\begin{aligned}
& R(\boldsymbol{H}, i)=\log _{2}\left[\operatorname{det}\left(\boldsymbol{I}_{N_{r}}+\frac{P_{r} \boldsymbol{H}(i) \boldsymbol{H}^{\mathrm{H}}(i)}{N_{r} \sigma_{w}^{2}}\right)\right] \\
& =\log _{2}\left[\operatorname{det}\left(\boldsymbol{I}_{N_{r}}+\frac{P_{t} \boldsymbol{H}(i) \boldsymbol{H}^{\mathrm{H}}(i)}{N_{r} \sigma_{w}^{2} C_{m m}\left(H_{U}^{2}+\|\boldsymbol{q}-\boldsymbol{u}\|^{2}\right)}\right)\right]
\end{aligned}
$$

where $\boldsymbol{H}(i)$ is an $\left(N_{r} \times K\right)$ channel matrix, and is obtained from $\boldsymbol{H}$ corresponding to the $i$-th antenna pattern activated for transmission. The random SSK makes each antenna pattern have the same probability $\frac{1}{|\mathcal{A}|}$ to transmit APM symbols, where $\mathcal{A}$ is a set containing all the antenna patterns used by the UAVBS with GrSM scheme. Taking account of all antenna patterns and channel statistics, the achievable ergodic rate of $R_{\text {signal }}$ can then be expressed as

$$
R_{\text {signal }}=E_{\boldsymbol{H}}\left\{\frac{1}{|\mathcal{A}|} \sum_{i \in \mathcal{A}} R(\boldsymbol{H}, i)\right\}
$$

where the expectation is taken with respect to the statistics of $H$.

Then, for the achievable rate of $R_{\text {spatial }}$, we assume that $x_{k}, k=1, \cdots, K$ are independent and is constrained to the same complex Gaussian distribution $x_{k} \sim C N\left(0, \sigma_{x}^{2}=1 / K\right)$. Then, the probability density function (PDF) of $x$ can be expressed as

$$
p(\boldsymbol{x})=\prod_{k=1}^{K} p\left(x_{k}\right)=\frac{1}{\left(\pi \sigma_{x}^{2}\right)^{K}} \exp \left[-\sum_{k=1}^{K} \frac{\left|x_{k}\right|^{2}}{\sigma_{x}^{2}}\right]
$$

where $|\cdot|$ denotes the modulus operator.

Hence, when the $i$-th, $i \in \mathcal{A}$ antenna pattern is activated, the received observations will also satisfy a complex Gaussian distribution. The PDF of the received signals can be written as

$$
\begin{gathered}
p(\boldsymbol{y} \mid i \in \mathcal{A})=\frac{1}{\pi^{N_{r}} \operatorname{det}\left(\boldsymbol{R}_{y \mid i}\right)} \exp \left(-\boldsymbol{y}^{\mathrm{H}} \boldsymbol{R}_{y \mid i}^{-1} \boldsymbol{y}\right) \\
=\frac{1}{\pi^{N_{r}} \operatorname{det}\left(P_{r} \boldsymbol{H}(i) \boldsymbol{C}_{x} \boldsymbol{H}^{H}(i)+\sigma_{w}^{2} \boldsymbol{I}_{N_{r}}\right)} \times \\
\quad \exp \left(-\boldsymbol{y}^{H}\left(P_{r} \boldsymbol{H}(i) \boldsymbol{C}_{x} \boldsymbol{H}^{H}(i)+\sigma_{w}^{2} \boldsymbol{I}_{N_{r}}\right)^{-1} \boldsymbol{y}\right)
\end{gathered}
$$

where $\boldsymbol{R}_{y \mid i}$ is the autocorrelation matrix of $\boldsymbol{y}$ with the $i$-th activation pattern, and $\boldsymbol{C}_{x}$ is the covariance matrix of $\boldsymbol{x}$, and is denoted as $\boldsymbol{C}_{x}=\boldsymbol{I}_{K} / K$.

Therefore, the rate achieved by the SSK can be calculated by the formula of [21]

$$
\begin{aligned}
R_{\text {spatial }} & =\max I(i \in \mathcal{A} ; \boldsymbol{y}) \\
& =\max _{p(i)} \iint p(i) p(\boldsymbol{y} \mid i) \log _{2}\left(\frac{p(\boldsymbol{y} \mid i)}{p(\boldsymbol{y})}\right) d \boldsymbol{y} \\
& =\frac{1}{|\mathcal{A}|} \sum_{i \in \mathcal{A}}\left[\int_{\boldsymbol{y}} p(\boldsymbol{y} \mid i) \log _{2} \frac{p(\boldsymbol{y} \mid i)}{p(\boldsymbol{y})} d \boldsymbol{y}\right]
\end{aligned}
$$

where $p(i)$ denotes the probability of the $i$-th antenna pattern activated, which is assumed to be uniform to maximize the rate of the SSK. Hence, we have $p(i)=1 /|\mathcal{A}|$. In (15), $p(\boldsymbol{y})=$ $\sum_{i \in \mathcal{A}} p(i) p(\boldsymbol{y} \mid i)$. 
Taking the flying area of the UAV-BS constraints into consideration, the problem can be formulated as,

$$
\max _{q_{x}, q_{y}} R_{\text {sum }}
$$

subject to :

$$
\begin{aligned}
& q_{x, l} \leq q_{x} \leq q_{x, u} \\
& q_{y, l} \leq q_{y} \leq q_{y, u}
\end{aligned}
$$

where $q_{x, l}$ and $q_{y, l}$ denote the minimum values of $q_{x}$ and $q_{y}$, respectively. $q_{x, u}$ and $q_{y, u}$ represent the maximum values of $q_{x}$ and $q_{y}$, respectively.

In this paper, the UAV-BS deployment intertwines with the GrSM, which makes the problem complicated to be solved. In this paper, we will rely on grid search algorithm to find the UAV-BS position that is capable of achieving a maximum sum rate.

\section{GRID SEARCH Algorithm AIDED OPTIMIZATION}

In this section, the UAV-BS flying area is divided into grids. The achievable sum rate on each grid point can be calculated separately in order to solve the optimal position of UAV-BS [14].

Given a fixed UAV-BS altitude, the flying area of UAVBS $\left[q_{x, l} q_{x, h}\right] \times\left[q_{y, l}, q_{y, h}\right]$ is divided into grids with a certain precision. Each point of the grid represents the projection coordinate on the ground for the UAV-BS. The step length of the $q_{x}$ and $q_{y}$ is denotes as $\triangle q_{x}$ and $\triangle q_{y}$. Thus, the coordinate of an arbitrary point of the grid can be expressed as,

$$
S_{g r i d}=\left[q_{x, l}+i_{x} \triangle q_{x}, q_{y, l}+i_{y} \triangle q_{y}\right]
$$

where $0 \leq i_{x} \leq \frac{q_{x, h}-q_{x, l}}{\triangle q_{x}}$ and $0 \leq i_{y} \leq \frac{q_{y, h}-q_{y, l}}{\triangle q_{y}}$.

\section{Simulations}

In this section, we evaluate the performance of the proposed mmWave-based UAV-BS deployment optimization with GrSM scheme. We consider an UAV-BS having $N_{t}$ transmit antennas serving ground user in mmWave frequencies. The ground user is randomly distributed in a rectangular area, which is also the hovering area of UAV-BS. The default parameters used are listed in Table I.

TABLE I

DefaUlt System PARAMETERS

\begin{tabular}{|l|l|l|}
\hline Parameter & Description & Value \\
\hline$H_{U}(\mathrm{~m})$ & Height of the UAV-BS & $100(\mathrm{~m})$ \\
$\sigma_{w}(\mathrm{dBm})$ & $\begin{array}{l}\text { Additive white complex Gaussian } \\
\text { noise }\end{array}$ & $-154(\mathrm{dBm})$ \\
$f_{m m}(\mathrm{GHz})$ & $\begin{array}{l}\text { mmWave frequency } \\
\beta\end{array}$ & correlation coefficient \\
$q_{x, l}(\mathrm{~m})$ & minimum values of $q_{x}$ & 0.6 \\
$q_{x, h}(\mathrm{~m})$ & maximum values of $q_{x}$ & $0(\mathrm{~m})$ \\
$q_{y, l}(\mathrm{~m})$ & minimum values of $q_{y}$ & $100(\mathrm{~m})$ \\
$q_{y, h}(\mathrm{~m})$ & maximum values of $q_{y}$ & $0(\mathrm{~m})$ \\
$\triangle q_{x}(\mathrm{~m})$ & the step length of $q_{x}$ & $100(\mathrm{~m})$ \\
$\triangle q_{y}(\mathrm{~m})$ & the step length of $q_{y}$ & $1(\mathrm{~m})$ \\
\hline
\end{tabular}

Fig. 2 depicts the sum rate performance of the UAV-BS deployment with GrSM scheme. In this simulation, the number

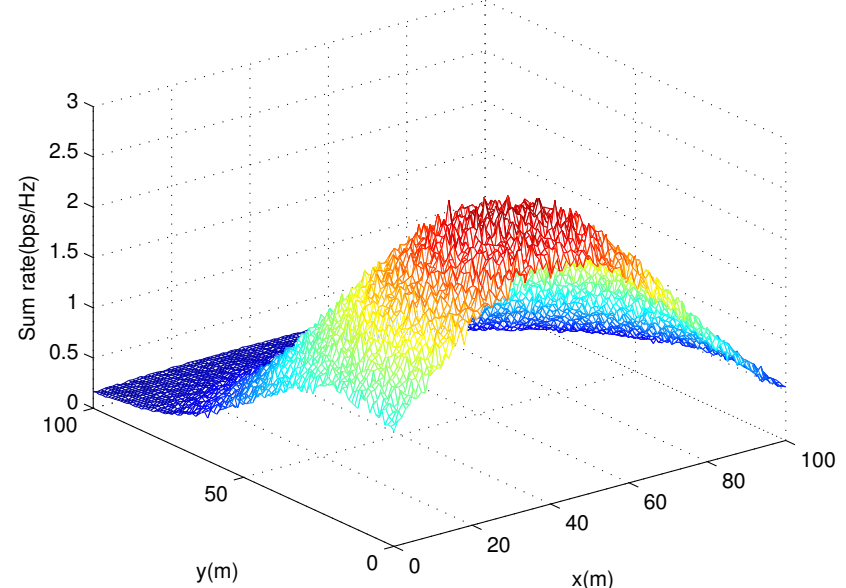

Fig. 2. Sum rate versus the coordinate of the UAV-BS utilizing GrSM scheme with $N_{t}=16, K=4, \beta=0.6$.

of transmit antennas is set as $N_{t}=16$, and they are divided into $K=4$ groups. The number of receive antennas for the ground user is set as $N_{r}=4$. The transmit power of the UAVBS is $30 \mathrm{dBm}$. Since the UAV-BS has fixed flying height, the $2 \mathrm{D}$ coordinate system is adopted to represent the projected coordinate of the UAV-BS. The UAV-BS flying area is divided into 10000 grids. The number of candidate UAV-BS positions in $x$ axis is 101. In $y$ axis, there also are 101 candidate UAV-BS positions. Thus, the number of projection positions belonging to the UAV-BS is 10201. In Fig. 2, the coordinate of the ground user is $[38,17]$. The optimal UAV-BS positions is $[37,18]$, and the achievable sum rate of the optimal UAV-BS location with GrSM scheme is 2.81430 bits/s/Hz. When $q_{y}$ is fixed at 0 , the sum rate performance increases upon increasing value of the $q_{x}$ coordinate until $q_{x}=37$, and then decreases upon increasing $x$ coordinate value. With fixed $q_{x}=0$, the sum rate curve improves a little. After $q_{y}=18$, the sum rate performance decreases fast, and then declines gradually with the increased value of the $q_{y}$ coordinate.

Fig. 3 shows the achievable sum rate performance of the optimal UAV-BS location with GrSM, the optimal UAV-BS location with GSM, the random UAV-BS location with GrSM, and the random UAV-BS location with GSM. The system parameters are set as $N_{t}=16, N_{r}=2, K=2$. The random coordinates of the UAV-BS is randomly generated in the flying area. The achievable sum rate performance of the optimal UAV-BS location outperforms that of the random UAV-BS location. It can be seen from Fig. 3 that the performance of GrSM scheme is superior to that of GSM scheme for both optimal UAV-BS locations and random UAV-BS locations, since GrSM takes account of the channel correlation. Additionally, at the target transmit power of $24 \mathrm{dBm}$, the attained gain of the optimal UAV-BS location with GrSM is about $0.2 \mathrm{bit} / \mathrm{s} / \mathrm{Hz}$ compared to the optimal UAV-BS location with GSM. Also, the random UAV-BS location with GrSM achieves 1.1603 


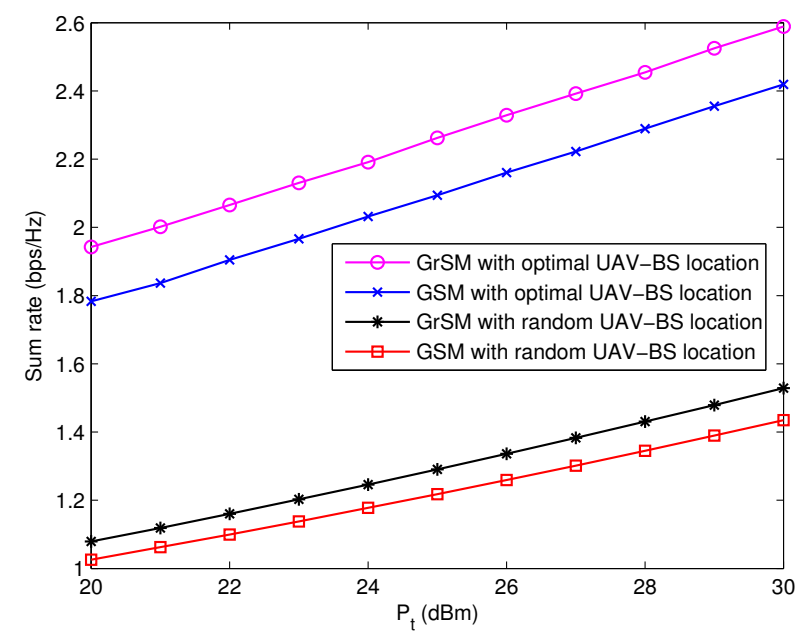

Fig. 3. Sum rate versus the transmit power for the optimal UAV-BS location with GrSM, the optimal UAV-BS location with GSM, the random UAV-BS location with GrSM, and the random UAV-BS location with GSM.

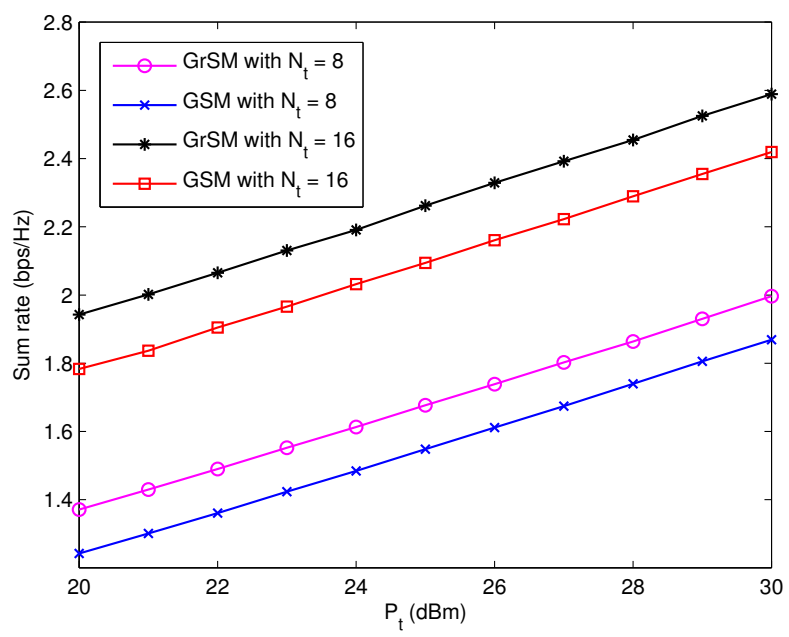

Fig. 4. Sum rate versus transmit power for the optimal UAV-BS location with GrSM and GSM for different number of transmit antennas with $N_{t}=$ $16 / 8, N_{r}=2, K=2$.

bits/s/Hz, while the random UAV-BS location with GSM is about 1.0996 bits/s/Hz.

In order to investigate the impact of the number of the transmit antenna, we compare the sum rate performance for optimal UAV-BS location with GrSM and GSM scheme in Fig. 4. In this case, $N_{r}=4, K=4$ are utilized for the optimal UAV-BS location with GrSM and GSM for different number of transmit antennas. As shown in Fig. 4, both the optimal UAV-BS location for GrSM and GSM scheme with $N_{t}=16$ achieve better sum rate performance compared to those with $N_{t}=8$. For GrSM scheme, the optimal UAV-BS location with $N_{t}=16$ achieves 1.9426 bits $/ \mathrm{s} / \mathrm{Hz}$ when $P_{t}=20 \mathrm{dBm}$, while that with $N_{t}=8$ reaches 1.371 bits/s/Hz. Moreover, for both $N_{t}=16$ and $N_{t}=8$, the sum rate performance of the optimal

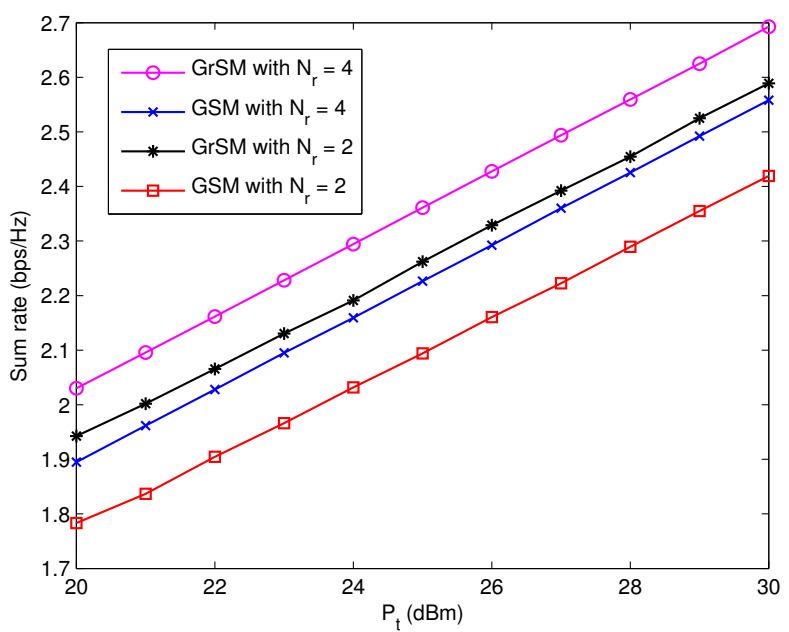

Fig. 5. Sum rate versus transmit power for the optimal UAV-BS location with GrSM and GSM for different number of receive antennas with $N_{t}=$ $16, N_{r}=2 / 4, K=4$.

UAV-BS location with GrSM outperforms that of the optimal UAV-BS with GSM.

In Fig. 5, we focus on the achievable sum rate performance for the optimal UAV-BS location with GrSM and GSM scheme for different number of the receive antennas. The setup is $N_{t}=16, N_{r}=2 / 4, K=4$. When $N_{r}=2$, the optimal UAVBS location with GrSM scheme has 0.11 bits/s/Hz improvement compared to the optimal UAV-BS location with GSM scheme at the target transmit power of $20 \mathrm{dBm}$. Both GrSM and GSM schemes in optimal UAV-BS locations with $N_{r}=4$ have superior sum rate performance than those with $N_{r}=2$. When the transmit power is $P_{t}=20 \mathrm{dBm}$, the optimal UAVBS location for GSM scheme with $N_{r}=4$ achieves 1.8926 bits/s/Hz. By contrast, given the same transmit power $P_{t}=20$ $\mathrm{dBm}$, the optimal UAV-BS location using GSM scheme with $N_{r}=2$ reaches $1.7832 \mathrm{bits} / \mathrm{s} / \mathrm{Hz}$.

\section{CONCLUSION}

In this paper, we investigated the optimization problem of maximizing the sum rate between the mmWave-based UAV-BS and the ground user. Meanwhile, the GrSM scheme is utilized to obtain extra throughput in spatial domain. In order to find the optimal location of the mmWave-based UAV-BS, we rely on the grid search algorithm. Simulation results demonstrated that the grid search algorithm could obtain the optimal deployment of the UAV-BS with GrSM scheme. Furthermore, our simulation results also showed that the mmWave-based UAV-BS relying on GrSM is capable of achieving a higher sum rate compared to that relying on GSM scheme.

\section{ACKNOWLEDGMENTS}

The financial support of the National Natural Science Foundation of China (NSFC): 61571401 and the Innovative Talent of Colleges and University of Henan Province under grant 18HASTIT021 are gratefully acknowledged. The work of G. Han was supported by the Science and Technology 
Development Project of Henan Province under Grant No. 202102210123.

\section{REFERENCES}

[1] J. Zhang, T. Chen, S. Zhong, J. Wang, W. Zhang, X. Zuo, R. G. Maunder, and L. Hanzo, "Aeronautical ad hoc networking for the internet-abovethe-clouds," Proc. IEEE, vol. 107, no. 5, pp. 868-911, 2019.

[2] Q. Wu, Y. Zeng, and R. Zhang, "Joint trajectory and communication design for multi-uav enabled wireless networks," IEEE Trans. Wireless Communi., vol. 17, no. 3, pp. 2109-2121, 2018.

[3] S. Rangan, T. S. Rappaport, and E. Erkip, "Millimeter-wave cellular wireless networks: Potentials and challenges," Proc. IEEE, vol. 102, no. 3, pp. 366-385, 2014

[4] C. Zhang, W. Zhang, W. Wang, L. Yang, and W. Zhang, "Research challenges and opportunities of UAV millimeter-wave communications," IEEE Wireless Communi., vol. 26, no. 1, pp. 58-62, 2019.

[5] R. Y. Mesleh, H. Haas, S. Sinanovic, W. A. Chang, and S. Yun, "Spatial modulation," IEEE Trans. Veh. Tech., vol. 57, no. 4, pp. 2228-2241, 2008.

[6] M. D. Renzo, H. Haas, A. Ghrayeb, S. Sugiura, and L. Hanzo, "Spatial Modulation for Generalized MIMO: Challenges, Opportunities, and Implementation," Proc. IEEE, vol. 102, no. 1, pp. 56-103, 2013.

[7] J. Wang, S. Jia, and J. Song, "Generalised spatial modulation system with multiple active transmit antennas and low complexity detection scheme," IEEE Trans. Wireless Communi., vol. 11, no. 4, pp. 16051615, 2012.

[8] X. Zuo, J. Zhang, X. Mu, and L.-L. Yang, "Channel correlation relied grouped spatial modulation for massive MIMO systems," IET Communi., vol. 14, no. 8, pp. 1241-1250, 2020.

[9] P. Ju, M. Zhang, X. Cheng, and C. X. Wang, "Generalized spatial modulation with transmit antenna grouping for correlated channels," in IEEE Int. Conf. Communi., pp. 1-6, Kuala Lumpur, Malaysia, May. 2016.

[10] Y. Cai, F. Cui, Q. Shi, M. Zhao, and G. Y. Li, "Dual-uav-enabled secure communications: Joint trajectory design and user scheduling," IEEE $J$. Sel. Areas Communi., vol. 36, no. 9, pp. 1972-1985, 2018.

[11] J. Lyu, Y. Zeng, R. Zhang, and T. J. Lim, "Placement optimization of uav-mounted mobile base stations," IEEE Communi. Lett., vol. 21, no. 3 , pp. 604-607, 2017.

[12] J. Sun and C. Masouros, "Deployment strategies of multiple aerial bss for user coverage and power efficiency maximization," IEEE Trans. Communi., vol. 67, no. 4, pp. 2981-2994, 2019.

[13] L. Zhu, J. Zhang, Z. Xiao, X. Cao, D. O. Wu, and X. Xia, "3-D beamforming for flexible coverage in millimeter-wave UAV communications," IEEE Wireless Communi. Lett., vol. 8, no. 3, pp. 837-840, 2019.

[14] Z. Xiao, H. Dong, L. Bai, D. O. Wu, and X. Xia, "Unmanned aerial vehicle base station (UAV-BS) deployment with millimeter-wave beamforming," IEEE Internet of Things J., vol. 7, no. 2, pp. 1336-1349, 2020.

[15] T. S. Rappaport, Y. Xing, G. R. MacCartney, A. F. Molisch, E. Mellios, and J. Zhang, "Overview of millimeter wave communications for fifthgeneration (5G) wireless networks-with a focus on propagation models," IEEE Trans. Antennas and Propagation, vol. 65, no. 12, pp. 6213-6230, 2017.

[16] T. S. Rappaport, S. Sun, R. Mayzus, H. Zhao, Y. Azar, K. Wang, G. N. Wong, J. K. Schulz, M. Samimi, and F. Gutierrez, "Millimeter wave mobile communications for $5 \mathrm{G}$ cellular: It will work!," IEEE Access, vol. 1, pp. 335-349, 2013.

[17] J. Zhang, S. Chen, X. Guo, J. Shi, and L. Hanzo, "Boosting fronthaul capacity: Global optimization of power sharing for centralized radio access network," IEEE Trans. Veh. Tech., vol. 68, no. 2, pp. 1916-1929, 2019.

[18] K. Kim, J. Lee, and H. Liu, "Spatial-correlation-based antenna grouping for MIMO systems," IEEE Trans. Veh. Tech., vol. 59, no. 6, pp. 2898$2905,2010$.

[19] A. Younis, R. Mesleh, M. D. Renzo, and H. Haas, "Generalised spatial modulation for large-scale MIMO," in 2014 22nd European Signal Proc. Conf. (EUSIPCO), pp. 346-350, Lisbon, Portugal, Nov. 2014.

[20] X. Wu, M. D. Renzo, and H. Haas, "Adaptive selection of antennas for optimum transmission in spatial modulation," IEEE Trans. Wireless Commun., vol. 14, no. 7, pp. 3630-3641, 2015.
[21] Y. Yang and B. Jiao, "Information-guided channel-hopping for high data rate wireless communication," IEEE Communi. Lett., vol. 12, no. 4, pp. 225-227, 2008.

[22] L.-L. Yang, Multicarrier communications. John Wiley \& Sons, 2009. 